\title{
On the Increasing Monotonicity of a Sequence Originating from Computation of the Probability of Intersecting between a Plane Couple and a Convex Body
}

\author{
BAI-NI GUO ${ }^{1, *}$, FENG QI ${ }^{2,3}$ \\ ${ }^{1}$ School of Mathematics and Informatics, Henan Polytechnic University, Jiaozuo City, Henan Province, China \\ ${ }^{2}$ College of Mathematics, Inner Mongolia University for Nationalities, Tongliao City, Inner Mongolia Autonomous Region, China \\ ${ }^{3}$ Department of Mathematics, College of Science, Tianjin Polytechnic University, Tianjin City, China \\ *Corresponding author: bai.ni.guo@gmail.com, bai.ni.guo@hotmail.com
}

Received December 25, 2014; Revised February 01, 2015; Accepted February 09, 2015

\begin{abstract}
In the paper, the authors confirm the increasing monotonicity of a sequence which originates from computation of the probability of intersecting between a plane couple and a convex body.
\end{abstract}

Keywords: increasing monotonicity, sequence, gamma function, ratio of two gamma functions, inequality, logarithmically completely monotonic function, probability of intersecting between a plane couple and a convex body

Cite This Article: BAI-NI GUO, and FENG QI, "On the Increasing Monotonicity of a Sequence Originating from Computation of the Probability of Intersecting between a Plane Couple and a Convex Body." Turkish Journal of Analysis and Number Theory, vol. 3, no. 1 (2015): 21-23. doi: 10.12691/tjant-3-1-5.

\section{Introduction}

On 19 December 2014, Mr. Yan-Zong Zhang, a mathematician in China, asked online a question: is the sequence

$$
p_{n}=\frac{n-1}{2}\left(\int_{0}^{\pi / 2} \sin ^{n-1} \theta d \theta\right)^{2}, n \in \mathbb{N}
$$

increasing? if not, can one take an example? where $\mathbb{N}$ denotes the set of all positive integers. On 20 December 2014, he told that this problem is needed by his teacher, Ms. Jun Jiang, and she said that this problem originates from computation of the probability of intersecting between a plane couple and a convex body in an unpublished paper.

The main of this paper is to give an affirmative answer to the above question.

Theorem 1.1. The sequence $p_{n}$ defined by (1.1) is strictly increasing.

\section{A Direct Proof of Theorem 1.1}

We firstly affirm the above question directly.

It is well known that the formula

$$
\int_{0}^{\pi / 2} \sin ^{n} x d x=\int_{0}^{\pi / 2} \cos ^{n} x d x=\frac{\sqrt{\pi} \Gamma((n+1) / 2)}{n \Gamma(n / 2)}, n \in \mathbb{N}
$$

is called the Wallis sine (cosine) formula, see [[9], Section 1.1.3], where

$$
\Gamma(z)=\int_{0}^{\infty} t^{z-1} e^{-t} d t, \quad \Re(z)>0
$$

is the classical Euler gamma function.

It is clear that $p_{1}=0$ and $p_{n}>0$ for $n>1$.

One may reformulate the sequence $p_{n}$ for $n>1$ in terms of the Euler gamma function $\Gamma(x)$ as

$$
q_{n}=\frac{n}{2}\left(\int_{0}^{\pi / 2} \sin ^{n} \theta d \theta\right)^{2}=\frac{n}{2}\left[\frac{\sqrt{\pi} \Gamma((n+1) / 2)}{n \Gamma(n / 2)}\right]^{2},
$$

for $n \in \mathbb{N}$. Hence, in order to make sure the increasing monotonicity of the sequences $p_{n}$ and $q_{n}$, it is sufficient to make clear the monotonicity property of the sequence

$$
Q_{n}=\frac{1}{n}\left[\frac{\Gamma((n+1) / 2)}{\Gamma(n / 2)}\right]^{2}, n \in \mathbb{N} \text {. }
$$

Taking the logarithm of $Q_{n}$ gives

$$
\ln Q_{n}=2\left[\ln \Gamma\left(\frac{n+1}{2}\right)-\ln \Gamma\left(\frac{n}{2}\right)\right]-\ln n \triangleq G_{n}
$$

and using the functional equation $\Gamma(x+1)=x \Gamma(x)$ leads to 


$$
\begin{aligned}
& G_{n+1}-G_{n} \\
= & 2\left[\ln \Gamma\left(\frac{n+2}{2}\right)+\ln \Gamma\left(\frac{n}{2}\right)-2 \ln \Gamma\left(\frac{n+1}{2}\right)\right]+\ln \frac{n}{n+1} \\
= & 2\left[\ln \frac{n}{2}+2 \ln \Gamma\left(\frac{n}{2}\right)-2 \ln \Gamma\left(\frac{n+1}{2}\right)\right]+\ln \frac{n}{n+1} \\
= & 4\left[\ln \Gamma\left(\frac{n}{2}\right)-\ln \Gamma\left(\frac{n+1}{2}\right)\right]+\ln \left[\frac{n}{n+1}\left(\frac{n}{2}\right)^{2}\right] \\
= & \ln \left[\frac{n}{n+1}\left(\frac{n}{2}\right)^{2}\right]-2\left(G_{n}+\ln n\right) \\
= & \ln \frac{n}{4(n+1)}-2 G_{n} .
\end{aligned}
$$

As a result, it suffices to prove $G_{n+1}-G_{n}>0$ which is equivalent to

$$
G_{n}=\ln \left\{\frac{1}{n}\left[\frac{\Gamma((n+1) / 2)}{\Gamma(n / 2)}\right]^{2}\right\}<\frac{1}{2} \ln \frac{n}{4(n+1)},
$$

that is,

$$
\left[\frac{\Gamma((n+1) / 2)}{\Gamma(n / 2)}\right]^{2}<\sqrt{\frac{n^{3}}{4(n+1)}}, \quad n \in \mathbb{N} .
$$

In [[7], p. 645], Gurland obtained that

$$
\left[\frac{\Gamma((n+1) / 2)}{\Gamma(n / 2)}\right]^{2}<\frac{n^{2}}{2 n+1}, \quad n \in \mathbb{N} .
$$

Later, Chu recovered the ineuqlaity (2.3) in [[4], Theorem 2]. Since

$$
\frac{n^{2}}{2 n+1}<\sqrt{\frac{n^{3}}{4(n+1)}}
$$

is equivalent to

$$
\left(\frac{n^{2}}{2 n+1}\right)^{2}<\frac{n^{3}}{4(n+1)},-\frac{n^{3}}{4(n+1)(2 n+1)^{2}}<0,
$$

the inequality (2.2) is valid. This implies that the sequence $Q_{n}$, and then the sequence $p_{n}$, is strictly increasing. The proof of Theorem 1.1 is complete.

\section{The First Indirect Proof of Theorem \\ 1.1}

Now we are in a position to give the first indirect proof of Theorem 1.1.

One may observe that the sequence $Q_{n}$ defined by (2.1) may be rearranged as

$$
Q_{n}=\frac{1}{n}\left[\frac{\Gamma((n+1) / 2)}{\Gamma(n / 2)}\right]^{1 /\left(\frac{n+1}{2}-\frac{n}{2}\right)}, \quad n \in \mathbb{N}
$$

$$
Q_{n}=\frac{1}{n} F_{1 / 2,0}\left(\frac{n}{2}\right),
$$

where

$$
F_{a, b}(x)=\frac{1}{x}\left[\frac{\Gamma(x+a)}{\Gamma(x+b)}\right]^{1 /(a-b)}, x>0 .
$$

Recall from $[1,11]$ that a positive function $f$ is said to be logarithmically completely monotonic on an interval $I$ if $f$ has derivatives of all orders on $I$ and its logarithm $\ln f$ satisfies $(-1)^{k}[\ln f(x)]^{(k)} \geq 0$ for all $k \in \mathbb{N}$ on $I$. For more information about the notion "logarithmically completely monotonic function", please refer to $[2,5,13,16,17,18]$ and closely related references therein.

In 1986, J. Bustoz and M. E. H. Ismail revealed in essence in [3] that

(1) the function

$$
f_{c}(x)=\frac{1}{(x+c)^{1 / 2}} \cdot \frac{\Gamma(x+1)}{\Gamma(x+1 / 2)},
$$

for $\quad x>\max \left\{-\frac{1}{2},-c\right\} \quad$ is logarithmically completely monotonic on $(-c, \infty)$ if $c \leq \frac{1}{4}$, so is the reciprocal of the function $f_{c}(x)$ on $\left[-\frac{1}{2}, \infty\right)$ if $c \geq \frac{1}{2}$

(2) the function

$$
f_{a, b ; c}(x)=(x+c)^{a-b} \frac{\Gamma(x+b)}{\Gamma(x+a)}
$$

for $1 \geq b-a>0$ is logarithmically completely monotonic on the interval $(\max \{-a,-c\}, \infty)$ if $c \leq \frac{a+b-1}{2}$, so is the reciprocal of the function $f_{a, b ; c}(x)$ on $(\max \{-b,-c\}, \infty)$ if $c \geq a$.

The logarithmically complete monotonicity of the function $f_{1 / 2}(x)$ and $f_{0,1 / 2 ; 0}(x)$ imply the strictly increasing monotonicity of the function $\left[F_{1 / 2,0}(x)\right]^{1 / 2}$ on $(0, \infty)$. Therefore, by the relation (3.1), the sequence $Q_{n}$, and then the sequence $p_{n}$, is strictly increasing. The proof of Theorem 1.1 is complete.

\section{The Second Indirect Proof of Theorem \\ 1.1}

Finally we give the second indirect proof of Theorem 1.1.

For real numbers $a, b$, and $c$, denote $\rho=\min \{a, b, c\}$ and let

$$
H_{a, b, c}(x)=(x+c)^{b-a} \frac{\Gamma(x+a)}{\Gamma(x+b)}, \quad x \in(-\rho, \infty) .
$$


In [[12], Theorem 1], Qi and Guo discovered the following necessary and sufficient conditions:

(1) the function $H_{a, b, c}(x)$ is logarithmically completely monotonic on $(-\rho, \infty)$ if and only if

$$
\begin{aligned}
& (a, b, c) \in D_{1}(a, b, c) \\
& \triangleq \\
& \quad\{(a, b, c):(b-a)(1-a-b+2 c) \geq 0\} \\
& \quad \cap\{(a, b, c):(b-a)(|a-b|-a-b+2 c) \geq 0\} \\
& \quad \backslash\{(a, b, c): a=c+1=b+1\} \\
& \quad \backslash\{(a, b, c): b=c+1=a+1\} ;
\end{aligned}
$$

(2) the function $H_{b, a, c}(x)$ is logarithmically completely monotonic on $(-\rho, \infty)$ if and only if

$$
\begin{aligned}
& (a, b, c) \in D_{2}(a, b, c) \\
& \triangleq \\
& \quad\{(a, b, c):(b-a)(1-a-b+2 c) \leq 0\} \\
& \quad\{(a, b, c):(b-a)(|a-b|-a-b+2 c) \leq 0\} \\
& \quad \backslash\{(a, b, c): b=c+1=a+1\} \\
& \quad \backslash\{(a, b, c): a=c+1=b+1\} .
\end{aligned}
$$

This means that the function

$$
H_{1 / 2,0 ; 0}(x)=\frac{1}{H_{0,1 / 2 ; 0}}=F_{1 / 2,0}(x)
$$

is strictly increasing on $(0, \infty)$, where $F_{1 / 2,0}(x)$ is defined by (3.2). As a result, by the relation (3.1), the sequence $Q_{n}$, and so the sequence $p_{n}$, is strictly increasing. The proof of Theorem 1.1 is complete.

Remark 4.1. The reciprocal of the sequence $p_{n}$ for $n \geq 2$ is a (logarithmically) completely monotonic sequence. For information on the definition of (logarithmically) completely monotonic sequences and related properties, please refer to closely related chapters in the books $[8,19]$.

Remark 4.2. By carefully reading the expository and survey articles $[9,10,14,15]$ and a large amount of references therein, one may deeply understand and extensively comprehend the spirit and essence of this paper.

Remark 4.3. This paper is a slightly revised version of the preprint [6].

\section{References}

[1] R. D. Atanassov and U. V. Tsoukrovski, Some properties of a class of logarithmically completely monotonic functions, C. R. Acad. Bulgare Sci. 41 (1988), no. 2, 21-23.

[2] C. Berg, Integral representation of some functions related to the gamma function, Mediterr. J. Math. 1 (2004), no. 4, 433-439.

[3] J. Bustoz and M. E. H. Ismail, On gamma function inequalities Math. Comp. 47 (1986), 659-667.

[4] J. T. Chu, A modified Wallis product and some applications, Amer. Math. Monthly 69 (1962), no. 5, 402-404.

[5] B.-N. Guo and F. Qi, A property of logarithmically absolutely monotonic functions and the logarithmically complete monotonicity of a power-exponential function, Politehn. Univ. Bucharest Sci. Bull. Ser. A Appl. Math. Phys. 72 (2010), no. 2, 21-30.

[6] B.-N. Guo and F. Qi, On the increasing monotonicity of a sequence, ResearchGate Dataset.

[7] J. Gurland, On Wallis' formula, Amer. Math. Monthly 63 (1956), no. 9, 643-645.

[8] D. S. Mitrinović, J. E. Pečarić, and A. M. Fink, Classical and New Inequalities in Analysis, Kluwer Academic Publishers, DordrechtBoston-London, 1993.

[9] F. Qi, Bounds for the ratio of two gamma functions, J. Inequal. Appl. 2010 (2010), Article ID 493058, 84 pages.

[10] F. Qi, Bounds for the ratio of two gamma functions: from Gautschi's and Kershaw's inequalities to complete monotonicity, Turkish J. Anal. Number Theory 2 (2014), no. 5, 152-164.

[11] F. Qi and C.-P. Chen, A complete monotonicity property of the gamma function, J. Math. Anal. Appl. 296 (2004), 603-607.

[12] F. Qi and B.-N. Guo, Wendel's and Gautschi's inequalities: Refinements, extensions, and a class of logarithmically completely monotonic functions, Appl. Math. Comput. 205 (2008), no. 1, 281290.

[13] F. Qi, S. Guo, and B.-N. Guo, Complete monotonicity of some functions involving polygamma functions, J. Comput. Appl. Math. 233 (2010), no. 9, 2149-2160.

[14] F. Qi and Q.-M. Luo, Bounds for the ratio of two gamma functions. from Wendel's asymptotic relation to Elezović-GiordanoPečarić's theorem, J. Inequal. Appl. 2013, 2013:542, 20 pages.

[15] F. Qi and Q.-M. Luo, Bounds for the ratio of two gamma functions-From Wendel's and related inequalities to logarithmically completely monotonic functions, Banach J. Math. Anal. 6 (2012), no. 2, 132-158.

[16] F. Qi, Q.-M. Luo, and B.-N. Guo, Complete monotonicity of a function involving the divided difference of digamma functions, Sci. China Math. 56 (2013), no. 11, 2315-2325.

[17] F. Qi, C.-F. Wei, and B.-N. Guo, Complete monotonicity of a function involving the ratio of gamma functions and applications, Banach J. Math. Anal. 6 (2012), no. 1, 35-44.

[18] R. L. Schilling, R. Song, and Z. Vondraček, Bernstein FunctionsTheory and Applications, 2nd ed., de Gruyter Studies in Mathematics 37, Walter de Gruyter, Berlin, Germany, 2012.

[19] D. V. Widder, The Laplace Transform, Princeton University Press, Princeton, 1946. 